\title{
छs \\ Doing science by waving hands: Talk, symbiotic gesture, and interaction with digital content as resources in student inquiry
}

\author{
Bor Gregorcic, ${ }^{1, *}$ Gorazd Planinsic, ${ }^{2}$ and Eugenia Etkina ${ }^{3}$ \\ ${ }^{1}$ Department of Physics and Astronomy, Uppsala University, Box 516, 75120 Uppsala, Sweden \\ ${ }^{2}$ Faculty for Mathematics and Physics, University of Ljubljana, Jadranska 19, 1000 Ljubljana, Slovenia \\ ${ }^{3}$ Graduate School of Education, Rutgers University, New Brunswick, New Jersey 08901, USA
}

(Received 4 April 2017; published 2 August 2017)

\begin{abstract}
In this paper, we investigate some of the ways in which students, when given the opportunity and an appropriate learning environment, spontaneously engage in collaborative inquiry. We studied small groups of high school students interacting around and with an interactive whiteboard equipped with Algodoo software, as they investigated orbital motion. Using multimodal discourse analysis, we found that in their discussions the students relied heavily on nonverbal meaning-making resources, most notably hand gestures and resources in the surrounding environment (items displayed on the interactive whiteboard). They juxtaposed talk with gestures and resources in the environment to communicate ideas that they initially were not able to express using words alone. By spontaneously recruiting and combining a diverse set of meaningmaking resources, the students were able to express relatively fluently complex ideas on a novel physics topic, and to engage in practices that resemble a scientific approach to exploration of new phenomena.
\end{abstract}

DOI: 10.1103/PhysRevPhysEducRes.13.020104

\section{INTRODUCTION}

Some approaches to instruction that engage students in practices characteristic of scientific inquiry (observing, hypothesizing, predicting, testing, etc.) take a relatively scripted form. That means they explicate the procedural steps students need to take in the process of inquiry. Indeed, this is often necessary, as students lack previous experience with such practices or are not yet able to spontaneously recruit and coordinate the procedural steps we want them to use as they learn to act like scientists. However, students sometimes also engage in practices that resemble scientific inquiry relatively spontaneously [1]. Gaining insight into such spontaneous emergence of sciencelike behavior should interest us, if we are to better understand and potentially leverage students' abilities for engaging in exploratory activities in productive ways [2]. In this paper, we shed light on some of the ways in which students can, if given the opportunity and an appropriate learning environment, spontaneously engage in collaborative inquiry, accompanied by discussion, which in a procedural sense resembles a genuine scientific approach to exploration of new phenomena. In particular, we pay attention to the way in which students express their ideas in a specific technology-supported learning environment on the topic of orbital motion.

\footnotetext{
*Corresponding author.

bor.gregorcic@physics.uu.se

Published by the American Physical Society under the terms of the Creative Commons Attribution 4.0 International license. Further distribution of this work must maintain attribution to the author(s) and the published article's title, journal citation, and DOI.
}

In the study described in this paper we show that students are able to express both conceptual and procedural ideas by juxtaposing different meaning-making resources. More specifically, they juxtapose talk with hand gestures that reference the underlying digital world projected on an interactive whiteboard (IWB) and with manipulations of the virtual world that is the focus of their inquiry. We present a series of illustrative excerpts from student group interactions that we have identified as bearing resemblance to scientific practices and interpret the students' use of a diverse set of meaning-making resources, ranging from talk, through gesture, to interaction with the digital environment. Our study shows how student nonverbal communication can play an active role in giving students not only access to new conceptual knowledge but can also help them in structuring their inquiry.

To lay the foundations for our analysis, we introduce and briefly summarize two bodies of research. The first is concerned with gesture and how it relates to other meaningmaking resources, most notably speech, but also to the physical and graphical structures in the environment. The second body of research is about learning to engage in scientific practices from a procedural point of view. Using a theoretical perspective grounded in these two bodies of research, the two research questions that we attempt to answer in this study are the following:

(1) What meaning-making resources (or a combination thereof) do students use in the studied interactive whiteboard-based learning environment?

(2) How do students employ the available meaningmaking resources to engage in discourse that resembles scientific practices? 
Our findings provide the interested researcher, teacher, and developer of curriculum materials with a nuanced insight into the interplay of student verbal and nonverbal meaning making as it takes place in a technology-enabled learning setting. We suggest that relatively common educational technology (the interactive whiteboard) can support inquiry-based learning environments that leverage typically relatively untapped learning potential. In our study, this potential reveals itself in the form of student representational ingenuity, as they seemingly effortlessly recruit meaning-making resources other than language. In turn, their use of these resources allowed them to spontaneously engage in the seeds of scientific practice.

We focus our attention on the procedural aspects of student engagement from the perspective of scientific practices, with educational technology at center stage. The interplay of student inquiry from a procedural perspective and multimodal communication in technology-rich learning environments is seldom addressed in existing research in physics education and more broadly. While there is existing research on gesture [3-7], learner-technology interaction $[8,9]$, and the learning of scientific practices $[10,11]$, the knowledge from this research is yet to be meaningfully integrated and grounded in concrete learning contexts that the physics education community would find relevant and interesting. We attempt to do this in this study. The patterns of student engagement and communication in the presence of modern technology should prove to be of increasing interest to physics educators at different levels, as the prevalence of interactive technologies in educational and everyday contexts rises. Teachers and curriculum developers may consider leveraging the possibilities of touch-screen technology for introducing more activities that make use of students' existing abilities for nonverbal meaning making. Researchers, on the other hand, may benefit by treating the interplay of students' gestures, talk, and interactions with the technology as an additional window into the process of learning.

\section{THEORETICAL FRAMEWORK}

\section{A. From talk to symbiotic gesture and meaning-making resources in the environment}

While much of research on discourse in learning settings is concerned with verbal and written communication, there has been a growing body of educational research that pays attention to hand gestures [12]. Gestures and their role and function in learning have been researched in experimental, quantitative studies (for examples from psychology, see Refs. $[13,14]$ ) and in interpretive, qualitative studies (for examples from science and mathematics education, see Refs. $[3,5,7,15,16])$.

Despite the diversity of investigative approaches and research questions, researchers seem to agree that gesture provides students with meaning-making possibilities that go beyond words, and that it provides insight into students' thought processes. Moreover, gesture even appears to be beneficial for the gesturing person's learning [17].

Interpretive studies suggest that student use of gestures during discussion helps them connect perceived, concrete features of physical phenomena with the abstract ideas and models of scientific disciplines [16]. Furthermore, and especially relevant for the purpose of this paper, gesturing in the presence of objects of interest, such as the experimental setup, can help students lower the cognitive load while they produce explanations in unfamiliar topics [12]. By serving as a bridge between functional manipulation of physical objects and verbal representations of such actions, gestures have been shown to help students express complex ideas even when they lack the required vocabulary $[7,18]$. Roth [12] even proposes that "when the classroom context supports the use of gestures students may develop scientific modes of discourse much more rapidly than when the context does not support the use of gestures" (p. 375). He suggests that gesture can also play a supportive role in shifting the form of student discourse, from merely observational statements to "theory talk" $[12,19]$.

We find two points that Roth and colleagues make in their extensive body of research particularly relevant for the purpose of this paper. They suggest that (a) gesture can promote the development of scientific language and discourse, and that (b) gesture should analytically be treated in concert with resources in the environment in which it is produced. The latter point has also been suggested by researchers in other fields, including anthropology [20], cognitive science [21], ${ }^{1}$ and semiotics [22].

Gestures that are "coupled" to the environment have been studied most extensively in anthropology and cognitive science, in order to shed light on the complexities of multimodal $^{2}$ communication in collaborative work [24-26]. Goodwin $[27,28]$ coined the phrase "environmentally coupled gesture" or "symbiotic gesture" to refer to hand movements, which are produced against an external, perceivable structure in the environment, and rely on the meaning provided by the environment to make full sense. For the purpose of this paper, we will refer to such hand movements as symbiotic gestures.

Examples of symbiotic gestures include hand movements performed by television weather forecasters, academics pointing to overhead slides, archeologists' gesturing when they discuss patterns in the soil [27,28], physicists

\footnotetext{
${ }^{1}$ Hutchins even frames these processes as indications of cognition itself being distributed between individuals and their surroundings.

${ }^{2}$ By multimodal, we mean communication that makes use of more than one meaning-making (semiotic) resource (e.g., talk, gesture, recruiting information in the surrounding environment). See Refs. [19,23] for a more in-depth discussion on the topic.
} 
discussing novel ideas against underlying graphical representations [29], and physics students and teachers collaboratively interpreting graphs [30] and diagrams [31].

Other classifications of gesture include the distinction between representational (gestures conveying some explicit meaning) and nonrepresentational gestures (gestures such as beats-rhythmical hand movement that accentuate speech) $[15,32,33]$. We limit our focus to representational gesture, which more explicitly carries physics meaning. Because it functions as representation, explicitly relating to physics content, we expect it to be the most interesting category of gesture for the physics education research community.

Furthermore, two dimensions of the so-called representational gestures will be of interest to us in this paper: iconic (gestures representing visual features, such as shapes, movement, etc.) and deictic (pointing gestures). Symbiotic gestures can have both a deictic and an iconic component, as we will also demonstrate in our study. They can be used to locate and draw attention to (deictic component), as well as describe and accentuate features in the environment (iconic component), or do both at the same time.

In summary, we take as our analytical point of departure the theoretical perspective proposed by Goodwin [26-28]. By studying human interaction and work situated in realworld contexts, he found that participants use different semiotic resources or "sign systems" to communicate complex ideas while they attend to a relevant setting, and that "none of these systems in isolation would be sufficient to construct the actions that the participants are pursuing. This suggests the importance of not focusing analysis exclusively on the properties of individual sign systems, but instead investigating the organisation of the ecology of sign system." (p. 36) [27].

\section{B. Investigative Science Learning Environment (ISLE) as a framework for interpreting the processes of students' inquiry}

In this study, we explore students' behavior in terms of procedural elements discerned from their discourse and interactions with the studied digital learning environment, and relate them to characteristic elements of scientific work. Below, we briefly summarize the second relevant body of research concerned with scientific practices.

While there is no consensus within science or the philosophy of science on what exactly the "scientific method" means [34-38], like Wittgenstein's family resemblance, there are certain elements that appear to be characteristic of scientific practice.

The Investigative Science Learning Environment (ISLE) framework $[39,40]$ is an instructional approach with the aim of teaching students the process of science and how to think like scientists. According to ISLE, the central elements that characterize scientific work are "empirical evidence, inductive and hypothetico-deductive reasoning, coherence of ideas, the testability of ideas, and collegiality" [39]. Within the ISLE framework, these characteristics are instantiated in the so-called ISLE cycle, consisting of five main steps that do not necessarily follow in a linear order: (a) observing phenomena and looking for patterns, (b) developing multiple explanations for these patterns, (c) designing new testing experiments and using these explanations to make predictions about their outcomes, (d) deciding whether the outcomes of the testing experiments are consistent with the predictions, and (e) revising the explanations if necessary [39].

Note that inviting students to generate several explanations for the same pattern and systematically test them in new experiments is a feature of ISLE that separates it from similar cycle-based approaches to curriculum frameworks.

Even though ISLE has been proposed as an instructional approach, we used its interpretation of the central aspects of science processes as an analytical lens to interpret what the students were communicating and doing. For another example of such use of ISLE, see Poklinek Čančula et al. [1].

\section{Bringing the theoretical perspectives together: The focus of our research}

The two bodies of research presented above serve as footholds for our analytical vantage point. Our research questions are as follows:

(1) What meaning-making resources (or a combination thereof) do students use in the interactive whiteboardbased learning environment?

Here, we have focused on how students make meaning by juxtaposing talk with gestures, how they ground their gestures in visually available resources, and how they interact with those resources, which are made available by the learning environment at hand.

(2) How do students employ the available meaningmaking resources to engage in discourse that resembles scientific practices?

More specifically, we want to see how students use language, gesture, and interaction with the environment to engage in elements of scientific practices, as they are conceptualized by the ISLE framework.

By addressing the above two questions, we wish to shed light on the process of the emergence of scientific (in a procedural sense) discourse among students in an inquirybased technology-supported collaborative learning setting on the topic of astronomy. In doing so, our work is positioned at the intersection of several areas (astronomy at high school level, computer-supported collaborative learning, multimodal discourse analysis, and the learning of scientific practices and procedures), which are underresearched, if not completely novel to the physics education research community. 


\section{OVERVIEW OF THE STUDY}

\section{A. Topic: Orbital motion}

The topic of orbital motion has appeared quite frequently in the media in the past few years, due to recent breakthroughs in astronomy. These include the successes of the European space agency's Rosetta mission [41], achieving a rendezvous of a manmade probe with a comet, the flyby of Pluto by NASA's New Horizons probe [42], and the more than two thousand exoplanets (planets orbiting distant stars) that were and are still being discovered by analyzing data from NASA's Kepler mission [43]. Even more recently, Batygin and Brown [44] analyzed data about the orbital motion of small distant objects in our own Solar System and proposed a hypothesis that there may be another, to this date unobserved, planet 10 times more massive than Earth, orbiting beyond Pluto, a very intriguing proposal, indeed. These examples may serve as illustrations that, though well understood, the topic of orbital motion is highly relevant and important in contemporary astronomy.

Traditionally, however, the treatment of orbital motion that goes beyond the basic example of circular orbits and recitation of Kepler's laws by the instructor has been largely absent in high school physics instruction, perhaps due to its detachment from everyday experience and the mathematical skills required to treat it analytically. One possible approach to the topic is through computer simulations. Yet, even if instructors use computer simulations to demonstrate how planets move around the Sun, there remains a challenge of how to actively engage students, rather than having them passively observe simulations in the role of demonstrations and listen to the teacher's accompanying explanations [45].

\section{B. Designed learning environment}

In the study at hand, students were engaged in a learning environment that we have designed as a part of a more extensive research project on the use of interactive whiteboards in physics instruction. As part of the project, we designed a learning environment that leverages the unique possibilities of IWB technology to allow students hands-on access to previously inaccessible topics [46]. One such case was the topic of orbital motion-Kepler's laws. We have combined the IWB with Algodoo software [47] to create an open-ended learning environment, in which students can create planets and throw them into orbits (or set their initial velocities by entering values of velocity components and/or angles) around a massive star.

The learning environment that we have designed to a large degree addresses the challenges mentioned in the previous section. It is designed to support student active and embodied engagement [48] with a topic that was traditionally out of experimental reach, and that is, at the same time, highly relevant for our understanding of the Universe around us. Furthermore, as we show in this paper, the environment can support students' engagement in practices that resemble scientific inquiry, though in an unorthodox environment - a computer-based world, where it is possible to observe and even engage with the motion of stellar objects that have been conveniently brought down to human spatial and temporal scales.

\section{Context and setting of the study}

The study we present in this paper took place in a secondary school for students of ages 14-19 in Slovenia. All participants were attending the second year of high school, were 15 or 16 years old, and had 3 years of regular physics lessons (two 45-minute lessons per week, 2 years in primary school, and 1 year in secondary school) prior to their participation in the study. Two weeks before the recorded session the students were introduced to dynamics of circular motion in class, and one week prior to the session they worked with Newton's law of universal gravitation. A few students already had some knowledge about Kepler's laws of orbital motion, but their familiarity with this topic varied considerably. In the Slovenian curriculum, Kepler's laws are a noncompulsory, optional topic. However, at the school where the study took place, they are traditionally included in the regular physics course plan. Typically, a lesson on Kepler's laws includes a lecture on the historical background of the study of orbital motion, stating the three laws and demonstrating a derivation of the third law for the special case of circular orbits. In recent years, computer visualizations have been used as demonstrations of the motion of planets and satellites.

All students involved in the study were familiar with the technical operation of IWBs. The school was fully equipped with IWBs and the students had opportunities to familiarize themselves with the IWB's basic functionality over a period of at least 6 months prior to our study. Students also reported experience of using touch-screen devices, such as smartphones and tablets, which are in many ways similar to IWBs [45].

A week prior to the recorded learning sessions one of the researchers, who was also in the role of the instructor in this study, introduced the participating students to the Algodoo software with a brief 15-minute outline of its basic functions. However, the participating students were not familiar with the possibilities of using Algodoo to explore astronomical phenomena until prior to the recording session. They were aware of most of its basic functionality, such as the various drawing and editing tools, as well as the possibility to play, pause, and undo a simulation.

At the beginning of the recording sessions each group of three students was given the same Algodoo scene to start with. The scene contained a circular yellow object on a dark background. The researcher, who sat at the back of the classroom during the student activity, gave a brief task to the students: "Explore the motion of smaller objects in the vicinity of this massive central object." The researcher also 
explained that students could create smaller objects by drawing them on the dark background. All three groups of students, consisting of 3 students each, quickly framed the situation as being astronomy related and identified the central yellow object as "a star" or "the Sun." The color scheme of the setup was intentionally selected to invoke such framing.

Since the three groups of students had different previous knowledge about the topic at hand, their explorations varied in pace and content. However, by the end of the activity, which lasted about 60 minutes, even the group with the least amount of previous knowledge about the orbital motion of planets (the group that we pay most attention to in this paper) was able to discern and qualitatively formulate Kepler's three laws of planetary motion. The other two groups, already having some previous knowledge about Kepler's laws, went on and explored in more detail the role of different kinematics parameters for the motion of planets about the Sun. The topic at hand and the possibilities for inquiry that the given technological setup afforded turned out to be very engaging and intriguing even for the more advanced students. For example, in addition to the qualitative and empirical investigations of the way planets move about the Sun, the advanced students posed questions such as the following: What is the minimum speed a planet needs to have in order to not crash into the Sun? Can the planet really fly off into infinity and never return? How will sudden changes in the planet's speed influence the shape of its orbit?

\section{Methods of data collection, selection, analysis, and presentation}

The selection of methods for data collection, presentation, and analysis was guided by the research focus. As we are interested in the multimodal nature of the student discourse, video and audio, instead of just audio, recording was a natural choice for the collection of data.

The data for this study consist of approximately 3 hours of video recordings. The video camera was placed on an elevated stand (about $2.5 \mathrm{~m}$ above the floor) about $4 \mathrm{~m}$ from the IWB, so that it did not disrupt the students' movement in front of the IWB. All participating students were in the frame of the camera from the knees up for most of the duration of the recording.

Since the three students involved in the learning activity and the researcher were the only people present in the room, the audio captured from the video camera was of sufficient clarity and quality to allow us to make written transcripts of the participants' speech. In the images presented in this paper the video frames have been cropped to better display the relevant details.

Next, we must comment on the selection of excerpts to be presented in this paper. The selected excerpts showcase student ingenuity and ability to express procedurally advanced ideas in a topic that is new to them, and for which they do not know the "proper" vocabulary. They first, and most importantly, exemplify the procedural steps that students were performing during their exploration. Secondly, while other cases of sciencelike procedural elements (hypothesizing, predicting, etc.) were discernable from the video data of all three groups, the selected excerpts showcase particularly well how nonverbal meaning-making resources, especially symbiotic gesture and interaction with the IWB, played into the procedural structure of student discourse.

All three groups engaged in extensive interaction with and gesturing against the IWB, as they proposed hypotheses and testing experiments, predicted outcomes, and produced explanations for observed phenomena. However, the descriptive nature of this paper required us to limit the selection of examples to a manageable and presentable sample size. For this reason, we have limited the selection to examples from two groups, but mostly to a set of episodes from the least advanced group of students.

Our methods of data analysis, drawing on the principles, commitments, and traditions of ethnomethodology and conversation analysis [49,50], and social semiotics [51], have also shaped the way we represent the video data to the reader. The way of presenting data in this paper was guided by the need to integrate what we see as the essential aspects of the verbal, gestural, and environmental modalities in a compact and easily interpretable representation [52]. Therefore, the figures in this paper should not be considered as raw data, but rather as interpretations of the original video recordings [53].

In order to make visible the interrelatedness and cooccurrence of speech, gesture, and the environment, the selected transcript excerpts and accompanying video screenshots are presented in parallel. To make student gestures and the movement of planets visible in the still video screenshots, we have drawn white and black arrows across the screenshots, mapping these movements in space. The white arrows represent the movement path of the students' hands when they gesture or interact with the IWB, and the black arrows represent the movement path of planets on the IWB screen. White dots represent locations where students pointed or held their hand for an extended period of time (more than a second, approximately). The underlined parts of the transcript text below the screenshot indicate the words that co-occurred with the student performing the gesture shown in the accompanying screenshot.

The selected episodes from the video data are thus presented using a combination of three channels.

(a) Transcription of the students' spoken utterances (translated from Slovenian to English for the purpose of this paper).

(b) Video screenshot of the students at the IWB as they speak the utterances transcribed in (a).

(c) Mapping of the movement of the speakers' hands in their environment and the movement of the planets on 
the IWB screen, using arrows [drawn across the screenshots (b)].

The presentation style that we use is a type of multimodal transcription [52,54]; we do not account for the pauses in speech, temporal overlaps of speakers' utterances, intonation, etc., since we do not see including them as being significant for portraying the content and structure of the selected episodes. ${ }^{3}$ However, we do, of course, recognize that our decision to use this particular transcription style is shaped by our theoretical perspectives on what is going on in the video recordings.

\section{ANALYSIS OF SELECTED EXCERPTS}

\section{A. Patterns of student investigation and interaction}

Students in all groups began to interact with the IWB immediately after they were given the task to explore the motion of smaller objects in the vicinity of the large central object. They had at their disposal one stylus, which they needed to control the IWB. They passed the stylus to each other spontaneously, as they were creating and manipulating the content of the IWB, interacting with the computer and with each other.

As we present the excerpts from the student activities, we make a case for what procedural role each of the excerpts played in the overarching narrative of student exploration of orbital motion. We look at how students used different resources to make meaning in each particular case and analyze how the use of these meaning-making resources played into students' inquiry.

\section{Performing observational experiments by physical interaction with the setting: Throwing planets into orbit}

An investigation of a phenomenon can begin in different ways. One that is particularly typical of human conduct is starting inquiry by physical input. Poking, disturbing, interfering with a system, or introducing into it new elements can result in changes to the behavior of a system, which can become the focus of observation and further investigation. This was also the case in the student activities analyzed in this paper. In terms of the ISLE framework [39], these actions are examples of performing an observational experiment. The aim of this step, as framed by ISLE, is to experience a phenomenon and observe it, followed by discernment of patterns (see Sec. IV A 2).

All of the recorded activities started with the students drawing new objects (planets to be) and letting them fall to the surface of the Sun due to gravitational attraction. It did

\footnotetext{
${ }^{3} \mathrm{Had}$ the participants in the selected excerpts been speaking in such a way that their utterances often overlapped, or if more than one student had used the IWB at the same time, we would most likely have selected a different transcription style, which could accommodate such intricacies of student interactions.
}

not take any of the groups a long time before they came up with the idea to send the planets into orbits around the Sun. The most direct way to do this is to simply grab the planet with the stylus, drag it, and retract the stylus away from the surface of the IWB, effectively performing a "touch-screen throw" (Fig. 1).

We now focus on group 1, in which none of the students knew about Kepler's laws before the session. In a matter of minutes, the students were engaging in the self-imposed "throwing challenge"- getting planets to move around the Sun in repeating orbits. The task is challenging because it is not immediately clear how one needs to throw a planet into an orbit that would meet two basic requirements: (1) the planet should not "run away" and (2) the planet should obviously not crash into the Sun. Much can be learned from the "throwing challenge" by either observing the motion of the planets after they are released or paying attention to the throwing action. However, we speculate that the greatest opportunity for learning lies in gaining the experience of performing the throwing action and reflecting on it from the first-person perspective, rather than merely observing others doing it.

By "throwing" planets into orbit, students engage their bodies while learning new physics. The act of throwing engages students in enacting the initial conditions of an orbit phenomena - the trajectory of an orbiting body will depend on its initial velocity (both direction and speed are important) and the point in space where the planet is "released." In this sense, the activity is conceptually congruent [48] with the targeted learning content—physics parameters that determine orbital motion.

In examples later in the paper, we show how the action of throwing becomes a shared experience to which students can relate in the further exploration of the topic. In what followed, students from group 1 reflected on the act of throwing and produced hand gestures that simulated the act of throwing (Sec. IVA 3), while another group even used the act of throwing itself as a communication resource during discussion (Sec. IV A 5). In this way, the action of throwing planted the seed for gestures that emerge later in the activity. This supports the findings of previous research on the relation of action and gesture [18,55].

However, first we take a closer look at how students formulated their observations of the outcomes of their initial investigation.

\section{Observing and describing patterns: Discovering Kepler's laws}

The next step in students' inquiry, as described and analyzed below, fits very nicely into the category of pattern recognition and representation. The step in the ISLE framework that corresponds to this is observing phenomena and looking for patterns [39].

When the students in group 1 got at least one planet to revolve around the Sun in a closed orbit (an elliptical orbit), 

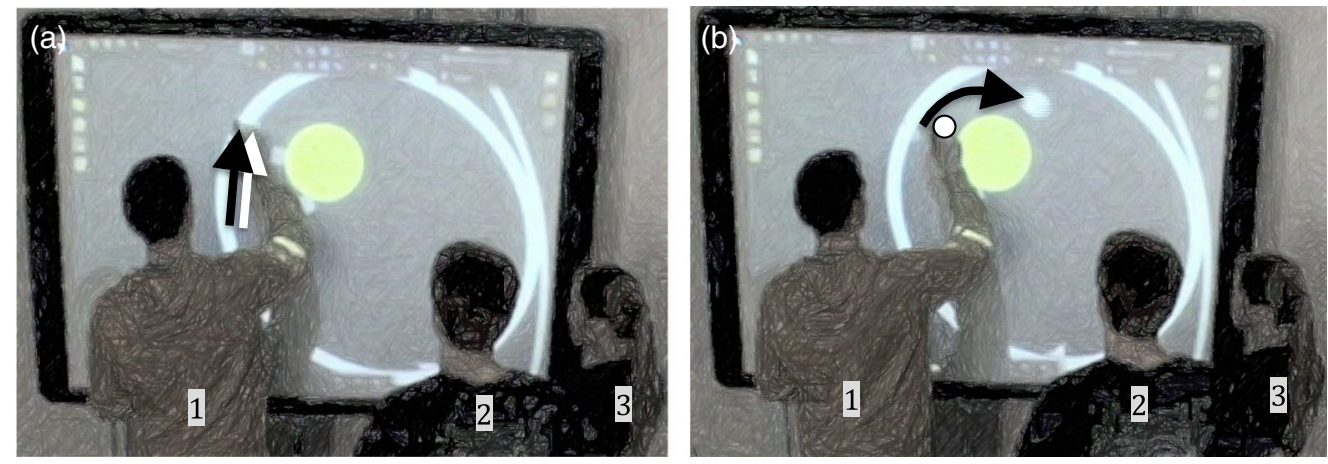

FIG. 1. A student throwing a planet into an orbit around the Sun. In (a) the white arrow represents the path that the student's hand traveled and the black arrow represents the path that the planet traveled. The paths overlap in (a) (the student was dragging the planet before he released it). In (b), the planet starts to orbit on a curved trajectory after the student released it by retracting the stylus from the IWB surface. The student's hand remains in the same place where he released the planet (the position of the hand is represented by the white dot).

all three groups of students spontaneously suggested that they want to trace the path of the planet on the background. The researcher, being well versed in the use of Algodoo, helped them to enable a tracer. In Algodoo, a tracer is a massless object that can be attached to other objects, which leaves a continuous trace of a preset duration on the background. The students' intention was to represent the movement trajectories of planets in a persistent way, allowing them to more easily examine the details of the geometrical shapes of the planets' trajectories. In most figures in this paper, the planets have tracers already attached to them, since the idea of attaching tracers to planets emerged quite early during all of the recorded activities.

The tracer example showcases how the students' resourcefulness can be leveraged if the instructor has sufficient knowledge about the possibilities of the tools the students are using. Note that the researcher did not prompt students to attach tracers to planets; he merely helped students follow through with their own ideas. Knowing Algodoo enabled him to act as a technical guide in situations where students produced useful ideas independently.

The students used their hands extensively to help them communicate their observations to each other. Their hand gestures were produced against the digital artifacts displayed on the board (planets, planet traces, the Sun, etc.). Verbal deictic terms like "this one" often accompanied gestures when students were referring to particular planets or their trajectories on the IWB screen. In Figs. 2 and 3, we pay specific attention to how students used symbiotic gestures in their efforts to describe their observations.

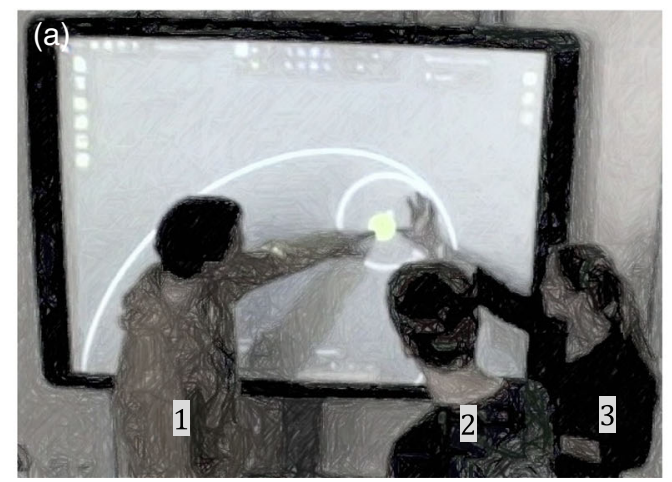

1: It looks like it is going in a circle. It's just that this one [pointing to the Sun] is not in the middle.

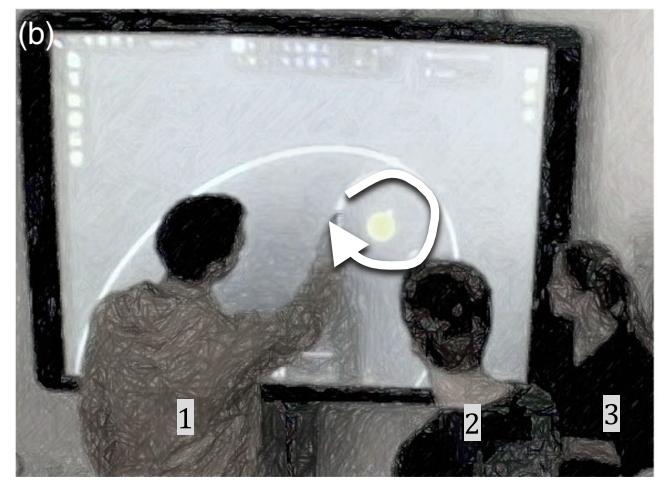

1: See how it goes [moving his hand]...

FIG. 2. In (a), two students (1 and 3) are pointing out that the Sun is not in the center of what looks like a circular-shaped orbit. Student 1 is pointing to the Sun, while formulating a verbal description of the visual characteristics of the orbits, as they are made visually available by tracers. Student 3 uses her hand to indicate the distance between the Sun and the orbit on one side, where the distance is the largest. In (b), student 1 uses another symbiotic gesture to describe the motion and orbital shape of the planet in a circlelike orbit. In this case, the symbiotic gesture allows the student to explicate the discerned features of the phenomena of interest by locating them in the setting itself, highlighting them, and thus bringing them to the attention of others. 
Students 1 and 3 in Fig. 2 are participating in the construction of shared meaning - they are expressing their observations by discerning observable features that seem relevant for the problem at hand. The distance-measuring gesture performed by student 3 [Fig. 2(a)] expresses an observable feature (a distance), further elaborating and supplementing student 1 pointing to the location of the Sun.

It is quite clear that it is simpler for students to express the small discrepancies between the observed and an ideal (perfectly centered and circular orbit) via a symbiotic gesture, combined with verbal deixis (this, that), than in a purely verbal form. This already points to one possible function that symbiotic gesture plays in the studied activity-it provides students a relatively fluent expressive pathway, freeing up cognitive resources.

Similarly, in Fig. 2(b), student 1 indicates the path of the planet across the trace that the planet had left on the background. This symbiotic gesture is iconic in two respects. Firstly, it represents the movement of the planet itself. This interpretation is supported by the accompanying student utterance ("how it moves") and is further indicated by the direction of the student's hand motion (clockwise, the same as the planet). Secondly, the gesture is also representative of the geometrical shape of the planet's trajectory or orbit-it represents the elliptic shape that the tracer leaves on the background. Once again, it serves to point out direct attention of the co-participants to a particular feature of the phenomenon of interest, effectively establishing a common focal point for the participants' attention and enabling collaborative meaning making.

In Fig. 3, student 1 further points out some of the variation in the patterns of different planets' orbital motion by making extensive use of symbiotic gestures.

It thus appears in this case that the symbiotic gesture plays an important role in the initial establishment of shared attention in the students' collaborative [see Fig. 2(a)] formulation of observations.
We see in excerpts shown in Figs. 2 and 3 that students have begun to recognize some of the central features of elliptic orbits of planets, and were able to express their observations using an interplay of symbiotic gestures and language.

First, they noticed and expressed their observation that the orbits are not necessarily perfect circles and that they can be more or less "circlelike" and proposed that the orbits may be elliptical, and second, they observed that the Sun is not located at the "center" of what they recognized to be the planet's elliptical orbit, but rather somewhere else. These observations can be considered to provide an empirical foundation for the more formally expressed Kepler's laws.

In fact, though not shown in this paper, the students in all three groups eventually successfully discovered on a qualitative level all three Kepler's laws. In addition to investigating some of the geometrical properties of orbits (as demonstrated above), they also found that planets move faster when they come closer to the Sun in their orbits (Kepler's second law) and that planets that are on average further away from the Sun take more time to complete their revolution (Kepler's third law). Furthermore, they were able to transduct [56] what they initially expressed using a juxtaposition of symbiotic gesture and talk into language alone when asked by the researcher to do so.

The examples above show how students were able to notice and express the patterns discerned using a wide range of meaning-making resources, from those provided by the software (tracers), made available by the students' environment, to symbiotic gestures, juxtaposed with speech. Note that students' talk contained almost no physics vocabulary associated with this topic (e.g., aphelion, eccentricity, focus). Rather, it was the symbiotic gesture that filled in the gaps and allowed students to express and communicate what they observed to each other and the researcher.

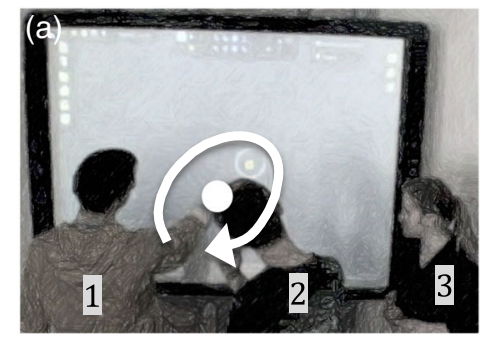

1: It doesn't have... if you look here in the middle [pointing] it is not the same circle all the time [moving his hand], like that one (referring to the smaller orbit).

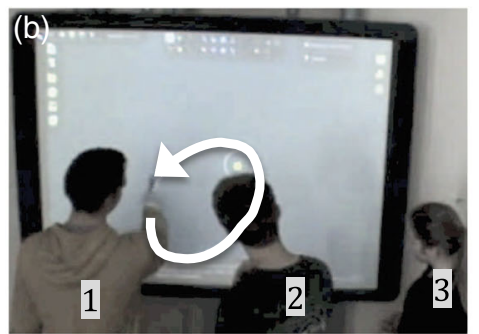

1: I mean, this looks like an ellipse [moving his hand]...

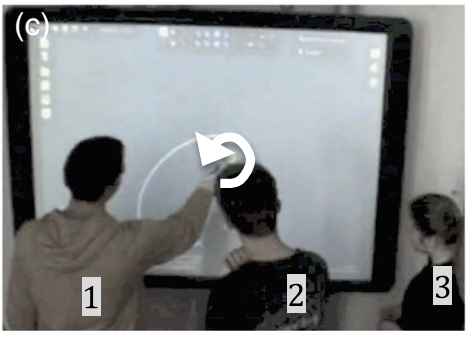

1: ....and this looks like a circle [moving his hand].

FIG. 3. In (a), student 1 is pointing to the "center" of the more pronounced (more eccentric) ellipse. In (b) and (c), he refers to the two differently shaped orbits, stating that "this one" (b) looks like an ellipse, and "this one" (c) looks like a circle. In this case (b),(c), the gestures represent the geometrical properties of the two orbits. Functionally, the symbiotic gestures shown here are employed by the student to express some discerned variation in the observed phenomena of interest. 
Drawing parallels to existing research of collaborative action in technology-rich environments, such as Hutchins's studies of pilots and naval officers [21,24], one can consider the student interactions in the above presented episodes as cases of distributed cognitive tasks. The vocabulary-heavy task of describing and conveying the patterns of orbital motion to fellow students is seemingly redistributed between the students' vocabulary, their gestures (most notably symbiotic gestures), interactions with the IWB, and the digital artifacts on the IWB themselves. In this sense, the IWB helps to redistribute some of the students' tasks into a wide palette of meaning-making resources. One of the most beneficial effects of such a distribution seems to be the decreased need for preexisting knowledge of specialized vocabulary. This means that students can approach the topic of instruction on a conceptual level even when they do not know the terms that would typically be used by expert physicists.

\section{Hypothesizing and predicting: "What determines a planet's orbit?'}

So far, we have seen how students have engaged with the IWB when "throwing" planets into orbits around the Sun (performing observational experiments in ISLE terms) and how they used their hand gestures in combination with speech and the environment (the IWB screen) to describe their observations (observing phenomena and looking for patterns in ISLE terms). In this section, we will give examples of the same group of students expressing their ideas and proposing ways of testing those ideas later in the session. The examples given below align with two main steps in the ISLE framework: developing explanations for the observed patterns and using these explanations to make predictions about the outcomes of testing experiments.

In Fig. 4, student 2 expressed an idea that a planet coming from far away and approaching the Sun, but just missing it on its approach, can orbit the Sun on a very elongated orbit. This clearly goes beyond pattern description and delves into the process of extrapolating observed patterns to come up with predictions. Furthermore, as we will see below, student 2 actually proposes a hypothesis about what determines the planet's motion in terms of orbit shape.

Once again, it is clear how symbiotic gestures performed by student 2 [Figs. 4(a) and 4(c)] and student 1 [Fig. 4(b)] allow students to construct shared meaning grounded in the perceivable materials on the IWB, and serve as a means of expressing ideas that are much harder to put into words than to enact. Effectively, symbiotic gestures allow the students to engage in speculation about the phenomena of interest in a clear and understandable way.

At a later point, the group was discussing what factors influence the shape of a planet's orbit, when student 2 proposed that the initial velocity with which a planet is launched (or thrown in our case) determines the shape of its orbit. This idea captures the essential principle required for the understanding of the kinematics of orbital motion. In cases where the mass of the star is much larger than that of the orbiting planet, and the distance between them is large compared to their radii, the planet's motion about the star will depend only on its initial velocity (speed and direction of motion). That means that if we know (or set) the planet's velocity at any point in space, this uniquely determines the planet's future motion trajectory and speed, as long as a third body, not part of the planet-star system, does not disturb it.

In Figs. 5-7 student 2 juxtaposes symbiotic gestures and talk to propose a testing experiment and explains what he expects to be the outcome of that experiment based on his hypothesis [39]; he suggests throwing a planet from a location in its existing orbit (which is almost circular) with a speed that is larger than the speed the planet had just prior to the imagined experiment.

The sequence in Figs. 5-7 is just over 6 sec long. Yet, it is extremely rich in terms of conceptual content and semiotic structure and plays a crucial role in the overarching narrative of the students' ongoing inquiry.

Similar to the previous examples, we can see that the symbiotic gestures in this sequence are mostly representations of planetary motion. However, there is one

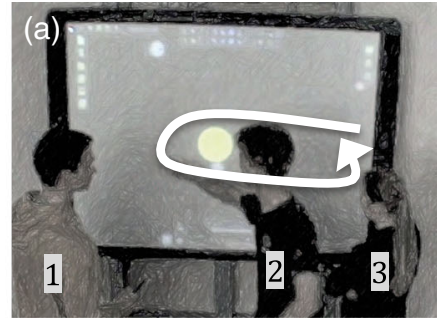

2: Can we put one here [start of the arrow] and have it come from very far away and directly inwards [moving his hand]?

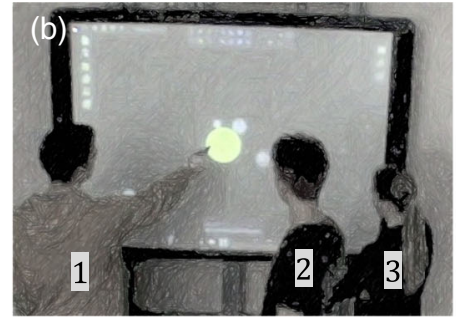

1: So, like the way this one came here? [pointing to a planet resting on the Sun]

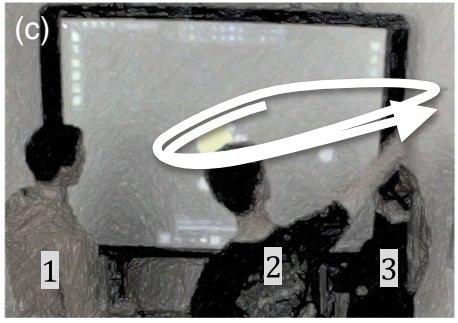

2: Yes, but so that it would miss it (the Sun) and go like this, far away again. [moving his hand]

FIG. 4. Student 2 suggests that if a planet started out far away from the Sun and moved towards it, but just missed it (a),(b), it would have a very elongated orbit (c). 


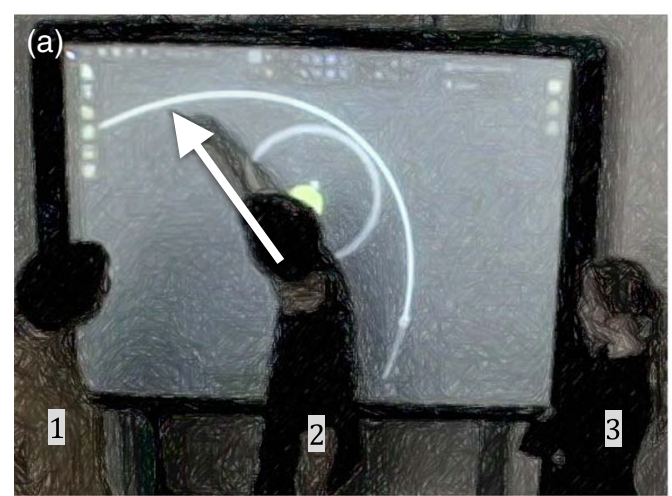

2: So if it went faster and we [moving his hand]...

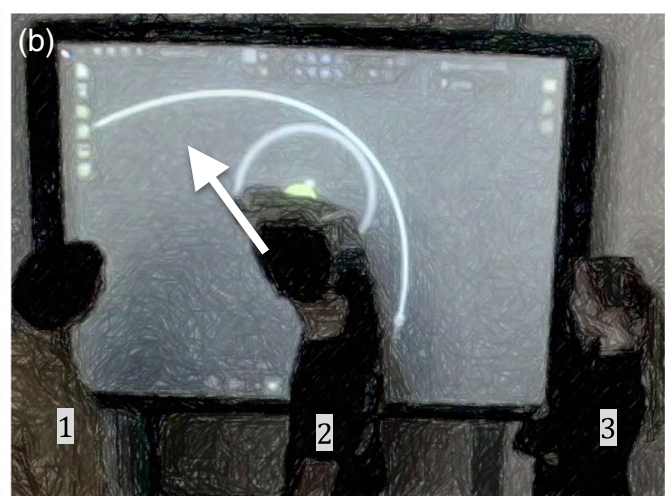

2: ... threw it from here like this [moving his hand]...

FIG. 5. The sequence of gestures is particularly interesting, because it mirrors students' previous interactions with the IWB. The gesture by student 2 in (a) represents the local movement of the planet. We can infer this from the accompanying student utterance. The gesture in (b), following directly the one in (a), simulates a "touch-screen throwing" motion with a finger-the student bends back his finger and flicks it as he retracts the hand from the screen surface (not clearly visible in the figure, but visible in the video).

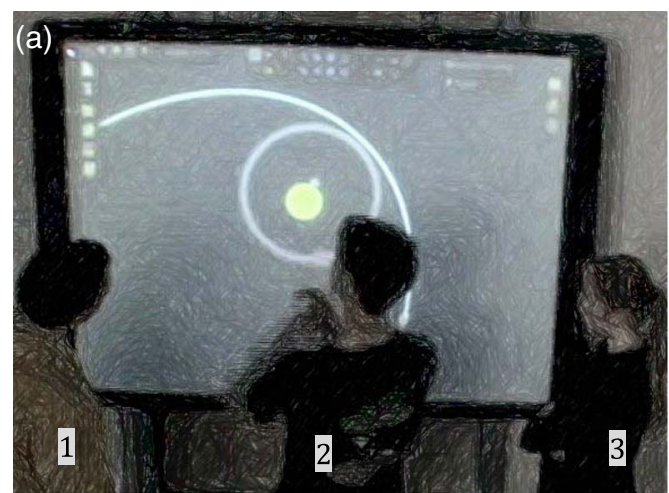

2: ...but not too fast...

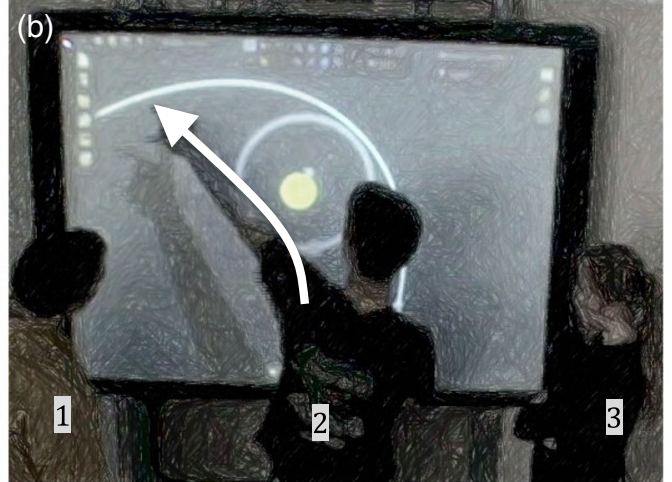

2: ... so it wouldn't fly away [moving his hand]...

FIG. 6. The student continues to elaborate on his idea. He changes the hand with which he gestures and swings it vigorously while saying the words "too fast." His finger in (b) is pointing towards a distant point out of the screen area (effectively extending the gesture space) to indicate that the planet, if thrown too fast, would fly far away. He supports this gesture with the words "fly away."
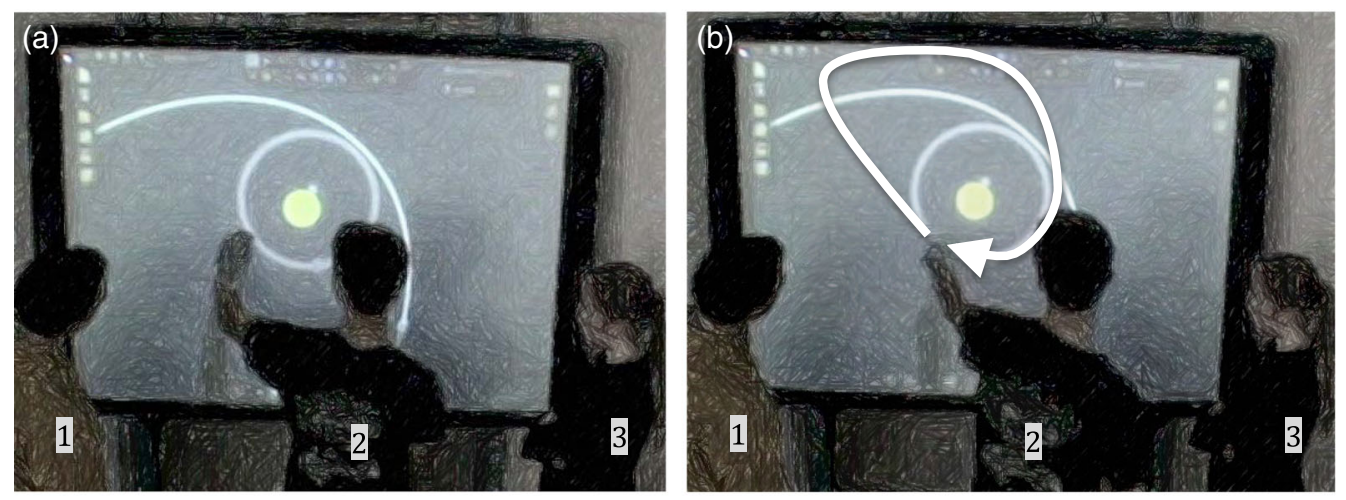

2: ...then I think it would come back in on an ellipse [moving his hand].

FIG. 7. Finally, student 2 gives a prediction of what he expects to happen in the "faster throw" experiment. He traces out the predicted path of the planet, starting and finishing it at the same point on the screen. The shape that he draws is quite irregular, not very similar to an ellipse, but this can be due to the lack of available screen area, the limited extent of the student's reach, and the student drawing the shape in a quick and rough style. Once again, the gesture can be seen as referring to both the planet motion and the geometrical shape of the orbit. 
particularly interesting gesture [Fig. 5(b)] that is different in this respect. The gesture represents the "throwing action." The student is bending his finger backwards as he simulates a throwing stroke and flicks the finger forward as he retracts the hand away from the screen. This gesture is interesting because it does not represent the motion of an object as an entity that is separate from the gesturing subject. In this case, the gesture is a simulation of an action performed by the gesturing subject himself. ${ }^{4}$ Furthermore, the gesture in Fig. 5(b) does not simulate an ordinary throwing action that one would perform throwing a real stone or a ball. It simulates a "touch-screen throw." This type of action is performed in interactions with touch-screen devices, such as tablets, smartphones, or IWBs. We can assume that the finger-flicking motion originates from student's previous experience with touch-screen devices. This serves as an illustration and a reminder that students actively draw on their physical experience of manipulation of and interaction with the world when they communicate through gesture $[18,55]$. The touch-screen throwing gesture is an indication that the experience on which the students can draw might also be actively generated by the students' interactions with modern technology, such as touch-screen interfaces. This observation offers us a tantalizing view of the potential of new technologies to help us leverage our capability for embodied learning. The ways of physical engagement with technology can be recruited to investigate and make meaning in new contexts. New technologies therefore have the potential to facilitate types of learning and problem solving that were previously not possible.

Finally, the sequence shown in Figs. 5-7 once again demonstrates the power and utility of symbiotic gestures for the communication of ideas. Gestures that are coordinated and coupled with the material setting (the IWB in this case) can be seen as a "broadband and high-speed connection" between the students' mental simulations and the shared communication space. Where language (especially the students' lack of vocabulary on the given topic, but also the nature of the observed phenomena) would in this case be an inconvenient bottleneck for the fluency of the student exchange of ideas, gestures, in juxtaposition with talk and semiotic resources in the environment, allow students to express their ideas in a fluent and efficient way. And once again, the sequence presented in this section demonstrates how such fluent exchange of ideas can be in service of what appears to be the seed of sciencelike inquiry.

\section{From symbiotic gesture to manipulation of the environment}

Thus far, we have shown how students can make use of symbiotic gestures to direct attention to specific features and establish common meaning. We now explore how students

\footnotetext{
${ }^{4}$ Such gestures have been classified as character viewpoint gestures [26].
}

engage with the environment, the digital world accessible through the IWB, in ways different than throwing. Here, we show that student interaction with the IWB was not only in service of performing observational experiments, but also served other purposes in the inquiry process as described by ISLE, such as performing testing experiments.

The following example (Fig. 8) shows students 1 and 3 making predictions of what they expect to happen if air resistance was suddenly introduced into the simulation. They made these predictions towards the end of their session, when they had already had some experience with the orbital motion of planets around the Sun.

Just before the excerpt in Fig. 8, student 3 suggested that the planets follow in their own path repeatedly (in exactly the same elliptical orbit again and again), because there is no resistance to impede their motion. The students produced the gestures depicted in Fig. 8 after the researcher asked them what would happen if there suddenly was air resistance.

Note that this kind of pointing has more than just a deictic role. It plays a central role for the iconicity of the gesture, as it is being used to depict the movement of the planet. Not having seen the planet actually spiral in yet, the gestures indicate that the students were running mental simulations of a hypothetical situation of planetary motion in novel circumstances (with imagined air resistance). Gestures can therefore serve as indicators that a mental simulation process (a thought experiment of sorts) is taking place. In fact, gesture has even been argued to play a role in cognition itself by allowing the students to offload some of their "thinking" onto their body's sensorimotor functions [7]. This can be interpreted as the student thinking through gesture, instead of it serving merely as an expressive mode. As the study at hand provides no further evidence to support a claim that gesture influences thinking, we will treat it as a mere possibility to be further explored by future research, although there is already some research that supports this hypothesis [57,58].

After a brief discussion, the students turned on air resistance in the Algodoo environment by pressing a dedicated button. The path that the tracer attached to the planet drew is shown in Fig. 9-the planet spiraled inwards toward the Sun. The students considered this to be in accordance with their predictions and were satisfied with the understanding that they were able to demonstrate by giving a prediction that matched the outcome of the testing experiment. Student 3 further noticed that the spiral trajectory was shaped in a particular way (the intermediate aphelia were aligned in the same direction, see Fig. 9), and once again expressed her observation using a juxtaposition of talk and symbiotic gesture (pointing out the alignment of intermediate aphelia), even though she did not know the expression "aphelion."

This example shows how the responsiveness of the environment in which the students investigate the 


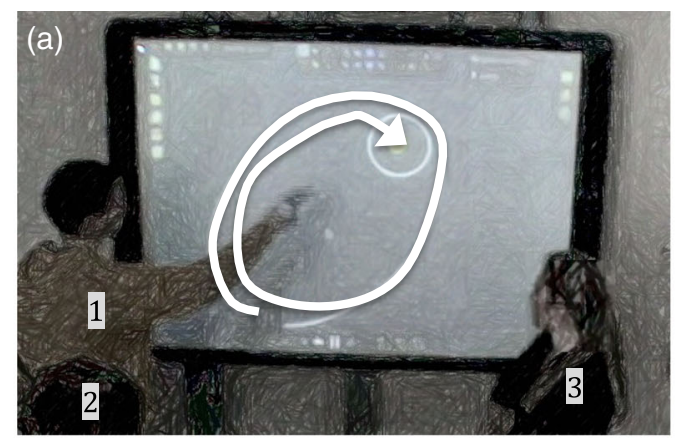

1: Then its ellipse would get smaller [moving his hand]

2: Its ellipse would slowly...

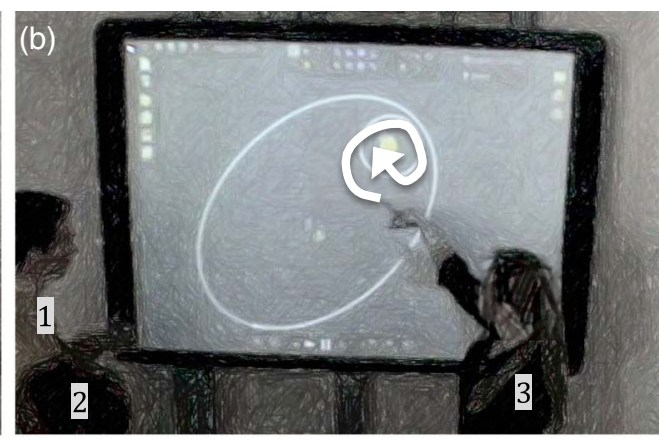

2: ....get smaller.

3: It would keep getting closer and closer [moving her hand].

FIG. 8. In the sequence shown in the figure, we can see a symbiotic gesture, produced by student 1 and its immediate repetition by student 3 . The gestures were produced in a rather quick manner, as rough illustrations of the type of motion that students 1 and 3 expected to see after they introduce air resistance into the simulation. The students did not move their hands in the plane of the IWB surface, but rather used their index finger to point to an imaginary point on the IWB surface, and in this way extended the effective spatial reach of their gestures. The arrow represents the position of the imaginary point of intersection between the direction of their pointing and the plane of the IWB screen.

phenomena of interest allows them to not only discuss hypothetical scenarios but also brings them into existence and tests their ramifications for the phenomena of interest. The case of introducing air resistance is particularly apt in demonstrating how the learning environment supported students' divergent thinking and discussion of "what if" questions. The instructor (the researcher in our case) was able to leverage students' ideas, and with a minor intervention in the form of a question, have them follow up their discussion with predictions (once again expressed by symbiotic gestures) and even with a realization of the imagined scenario in the form of a testing experiment. The flexibility and responsiveness of the environment therefore allowed and, in combination with appropriate framing of

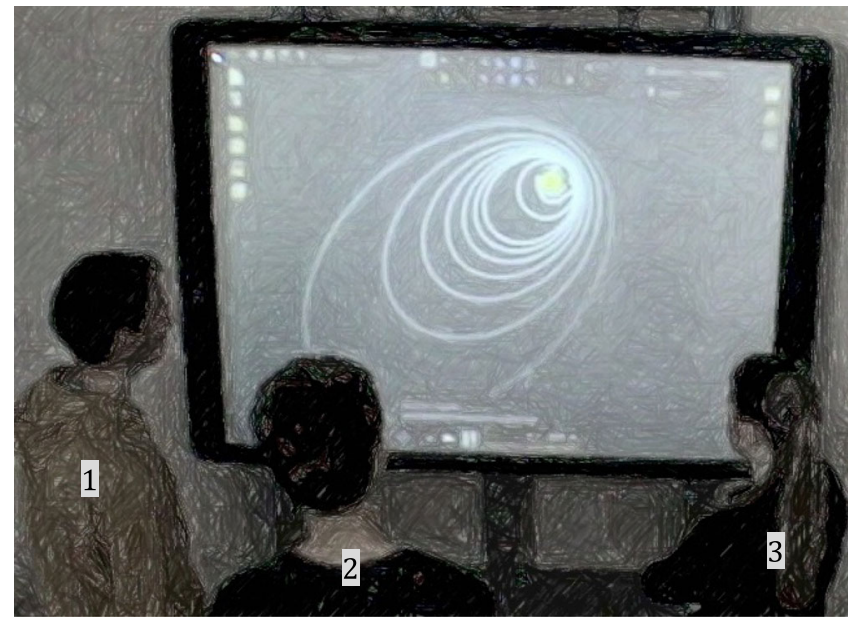

FIG. 9. The outcome of the experiment, where air resistance was introduced into the simulation of planets orbiting the Sun. The planets, as the students predicted, spiraled inwards towards the Sun. The spiral is shown. the task, also encouraged students to try things that they could not do in real-world settings. Such interactions with the IWB content gave rise to new observations, in turn fueling the cycle of inquiry.

\section{Telling by doing: "Throwing" as a communicative act}

The last example in this paper shows a sequence of interactions between students from group 2 . The sequence demonstrates how students can integrate interaction with the IWB into their discussion in a fluid way, so that interaction with the IWB itself becomes a meaning-making resource, similar to gesture and speech. In a sense, we can see that there is an apparent continuity and fluency between symbiotic gesture such as that shown in Fig. 5 (throwing a planet) and actual throwing of a planet, to communicate a certain message. In addition to the semiotic richness, the sequence also exhibits characteristics of sciencelike discourse, namely, a debate centered around two competing hypotheses.

The following sequence (Figs. 10, 11, and 12) shows two students engaging in a discussion about whether a planet, independent of its initial velocity, always comes back (formally, this is a question about the existence of the so-called escape velocity). The discussion started with one of the students proposing a requirement for a planet to start orbiting around the Sun (Fig. 10).

The following snippet (Fig. 11) happened right after the clash of ideas shown in Fig. 10. Student 4 turned towards the IWB, quickly drew a new planet, and threw it towards the right-hand side of the IWB with a very high speed. Student 4 made use of the responsiveness of the digital environment to create a desired situation instead of just talking (or gesturing) about it. This way, without saying a 


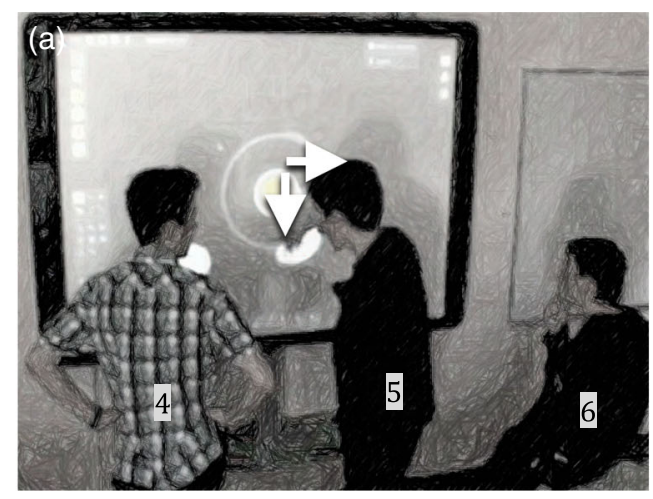

5: If it makes this distance [moving the hand horizontally] before it makes this distance [moving the hand vertically], then it will start circling.

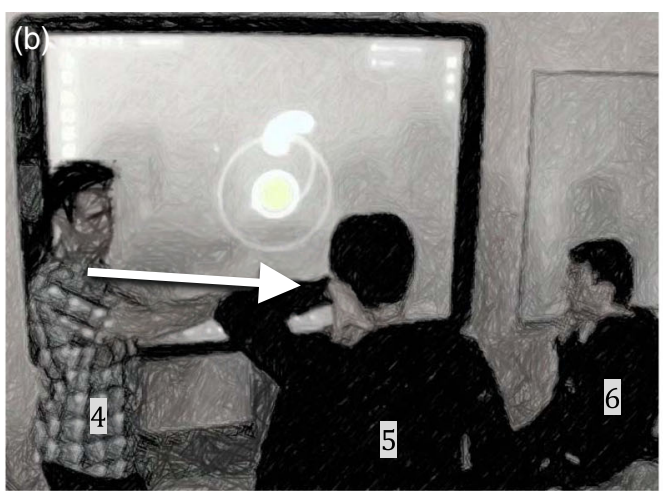

4: Yeah, but it shouldn't go too fast, so it doesn't get thrown out [moving his hand].

5: But it is circling, no matter how far you throw it. How to make it really circular is another question...

FIG. 10. Student 5 proposes an explanation for how the planet starts orbiting around the Sun (the planet moves forward faster than it moves towards the Sun). To that, student 4 replies that the planet also should not move too fast, otherwise it will fly away and not return. The students do not agree on whether there is such an escape velocity. Student 5 claims that the planets will always return, but their orbits do not necessarily have a perfect circular shape.

word, he was able to realize his idea of an experiment, with the intention of convincing the other student.

In this case, the student's interactions with the IWB can be seen as meaning-making actions. The spontaneity with which students were able to operate the equipment allowed them to use not only gestures that mimic action (like in Fig. 5, for example) but action itself with the purpose of communicating their ideas. The fluency and ease with which student 4 in Fig. 11 had drawn the planet and performed the throwing action can be compared to that of the gestures shown in Figs. 5-7. Interestingly, Adams [59] found that one of the reasons why students use gestures when using the simulation is that they are unable to quickly cause the simulation to provide the visualization that would match their talk. The example we give in Fig. 11 suggests that if the interface allows students a very direct and efficient way of engaging with the simulation, students can spontaneously recruit the available resources in the simulation software and integrate them into the ecology of meaning-making resources that they use in their discussion. In this way they can, as shown above, produce a testing experiment (experiment where different hypotheses can be judged based on the outcome) as an integrated part of their multimodal discourse. The sequence in Figs. 10, 11, and 12 thus not only showcases students' resourcefulness and ingenuity when they work with highly responsive digital tools, but also gives us a glimpse of an exciting and engaging (for the students) discussion centered around two competing hypotheses. The very process of such discussion is potentially a productive breeding ground for the emergence of sciencelike practices.
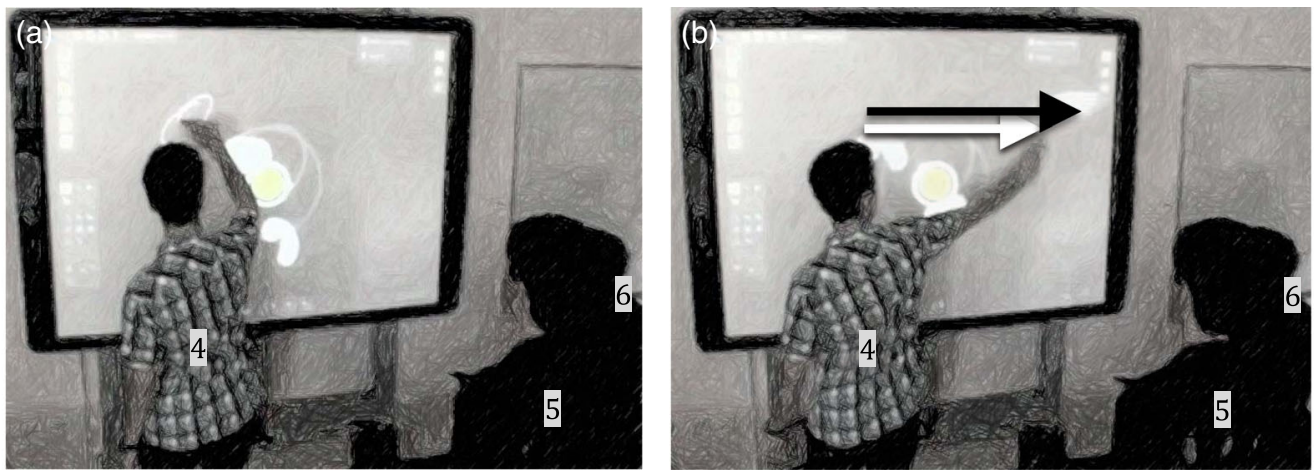

FIG. 11. Student 4 turns to the IWB, quickly draws a new planet, grabs it, and throws it with a very large velocity towards the righthand side of the screen. 


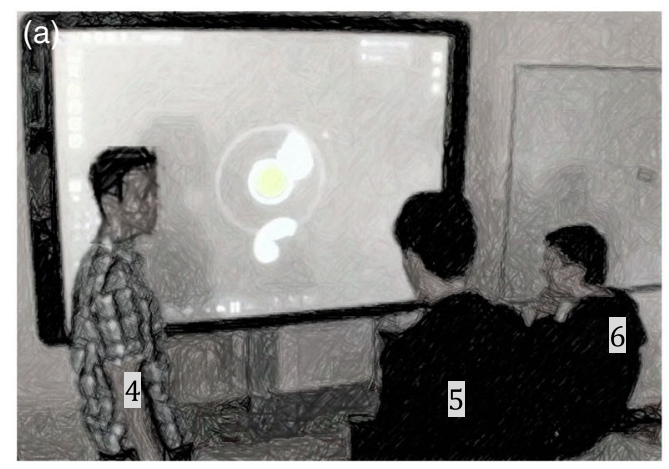

4: Now I've used a larger velocity than it would need to...

6: (laughing)

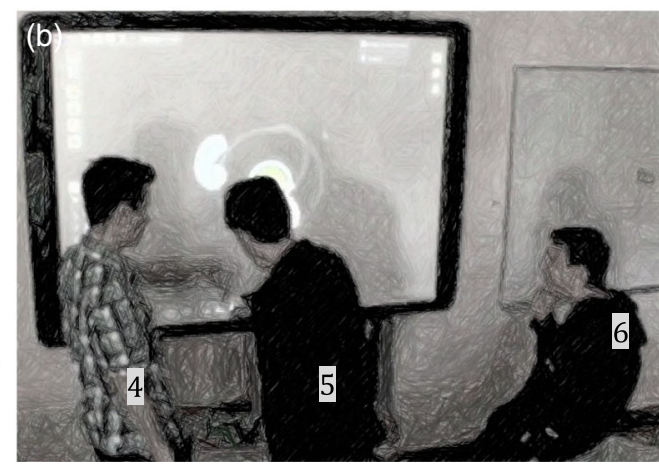

5: Exactly, now speed up the time a million times, and it will come back.

6: (laughing)

FIG. 12. After the throw, student 5 responded by proposing his own idea of how to observe the outcome of this experiment that would, as he believed, support his own argument that a planet would return after a long time. He knew that the simulation speed could be adjusted and suggested that they increase its speed to avoid waiting a long time before the planet would supposedly return.

\section{DISCUSSION}

\section{A. Answering the research questions}

The presented analysis of student interactions in the studied learning environment allows us to answer our research questions.

(1) What meaning-making resources (or a combination thereof) do students use in the interactive whiteboard-based learning environment?

The analysis of the cases presented in this paper clearly shows that students rely heavily on meaning-making resources other than talk, as they engage in the studied activities. In addition to talk, students make use of hand gestures and juxtapose them with talk and features in the environment (what is being displayed on the IWB), producing so-called symbiotic gestures [27], which allow them to express nuances of meaning that are difficult to put into words (see Secs. IV A 2-IV A 5). Furthermore, they take advantage of the intuitive manipulability of the technological setup (the combination of the IWB with Algodoo) to manipulate on-screen content and produce virtual scenarios as they discuss ideas that emerged during their activities (see Secs. IVA 4 and IVA 5).

(2) How do students employ the available meaningmaking resources to engage in discourse that resembles scientific practices?

We have used the ISLE framework [39] to identify elements of sciencelike practices in student activities. We have shown examples of how students juxtapose talk with symbiotic gestures in describing observed patterns (Secs. IVA 2 and IVA 4), proposing testing experiments, and making predictions about their outcomes (Secs. IV A 3IV A 5). Furthermore, students also manipulate the environment itself when performing observational experiments (Sec. IV A 1), customizing simulation parameters to perform testing experiments (Sec. IV A 4) and perform on-the-spot testing experiments inspired by the topic of discussion at that very moment (Secs. IVA 4 and IVA 5). What is most interesting about these findings is that the observed patterns of student interaction and discourse often emerged without specific prompting (the exception being Sec. IV A 4). This indicates that, given the right conditions, students can in fact spontaneously engage in what appears to be the seed of scientific practices.

\section{B. Implications for instruction}

Based on these findings, we propose that visually accessible and easily manipulable digital environments, when used in collaborative open-ended inquiry-based activities, can support the emergence of students' sciencelike behavior. They do this by allowing students to recruit a variety of meaning-making resources that are typically not considered to be the central means of communication in the physics classroom.

In practice, teachers can place more emphasis on encouraging students to make use of their hands during discussion, especially when they are yet to develop a specialized vocabulary in the topic. Conceptual and procedural ideas may initially manifest themselves through "hand waving" before they can be wrapped into expert vocabulary. Naturally, learning the discipline's vocabulary is a part of moving towards expertise. However, for a teacher, attaching words to concepts that students have learned and even expressed using other means of communication will be the easy part.

Our study also points to a powerful role educational technology can play in physics instruction. Rather than connecting physically isolated individuals over the Web, which is the way in which many computer-supported collaborative learning environments tend to function, technology can bring together individual learners in a genuinely 
shared physical space. The IWB, as it is being used in our study, functions as a connecting social hub against which student embodied engagement obtains a meaningful function in the process of learning to act like a scientist. Such use of technology maintains personal human contact and face-to-face exchange of ideas as its central tenet. We can speculate that in addition to talk and gesture, the highly nuanced expressive possibilities of body posture, gaze, facial expression, speech intonation, etc. provide students with an even more versatile and flexible set of meaningmaking resources. If teachers want to capitalize on the potential of technology, as it is discussed in this paper, they should use the technology to physically bring students together.

Implementing the presented learning materials in different settings, such as large classrooms, and in different physics topics will surely be accompanied by challenges. Scaling the activities up in terms of classroom size and maintaining their character can be difficult. Further research is needed to explore different ways of bringing such activities into large classrooms and addressing the accompanying challenges. A possible way of bringing the described activities into a larger classroom is migrating them from an IWB to a larger number of tablets equipped with the same software. Where teachers have tablets at their disposal, we suggest they put students into pairs to avoid overcrowding around each tablet and to maximize the possibility for each student to engage with the simulation.

Using a single IWB in a large classroom setting will most likely result in a different character of exploration [45]. Engaging students in whole-classroom discussion and investigation requires the teacher to take on a more central role, actively delegating tasks between students and leading the discussion [45]. In any case, it is important that the teacher is well prepared to operate the combination of software and hardware, in order to be able to help students with technical advice and to help them stay "on track" [45].

While we have invested significant time and thought into preparing the digital materials that were used in this study, Algodoo and the IWB, as well as other software and hardware combinations, may be able to foster similar types of student interactions in other topics. Algodoo offers possibilities for open-ended inquiry-based activities on a diverse set of topics [47], from geometrical optics to the study of gases and crystals at microscopic scale. Educational simulations such as PhET [60] and games that engage learners kinesthetically $[61,62]$ may be another source of inspiration.

\section{Future research}

Further research is needed before we can make any broader generalizations about the role and importance of particular features of instructional design, instructor interventions, and contextual factors in bringing about student behavior that mirrors scientific practices in the way that we have observed in this study.

In terms of the ISLE framework, our study therefore primarily stands for observation and description of patterns. As such, it allows hypothesis generation, but not yet rigorous hypothesis testing. It provides us with valuable insight into the ways students organize inquiry and meaning making in novel settings. They do it by spontaneously recruiting their bodies and digital materials at their disposal, juxtaposing them with talk and each other, to express what they struggle to put into words alone.

One hypothesis to be further tested by future research is that the combination of framing the activity as open-ended inquiry, limiting the scope of potential exploration by defining the topic of interest, and providing the students with a visually accessible and intuitively manipulable environment is what allows them to engage in the productive behaviors similar to those described in this paper. This implies that similar processes may take place in other settings, including inquiry-based laboratory sessions, where students interact with physical equipment and discuss experimental procedures. We hope that future research will further draw on multimodal discourse analysis and look at collaborative active learning settings, where students congregate and interact in the presence of and with materials, be they digital or physical.

\section{ACKNOWLEDGMENTS}

The authors acknowledge financial support from the Swedish Research Council (Project No. 2016-04113) and the Slovenian Research Agency (research core funding No. P1-0060).
[1] M. Poklinek Čančula, G. Planinšič, and E. Etkina, Analyzing patterns in experts' approaches to solving experimental problems, Am. J. Phys. 83, 366 (2015).

[2] D. Hammer and E. van Zee, Seeing the Science in Children's Thinking: Case Studies of Student Inquiry in Physical Science (Heinemann, Portsmouth, NH, 2006).
[3] B. W. Dreyfus, A. Gupta, and E. F. Redish, Applying conceptual blending to model coordinated use of multiple ontological metaphors, Int. J. Sci. Educ. 37, 812 (2015).

[4] E. A. Chase and M. C. Wittmann, AIP Conf. Proc. 1513, 94 (2013). 
[5] R. E. Scherr, Gesture analysis for physics education researchers, Phys. Rev. ST Phys. Educ. Res. 4, 010101 (2008).

[6] W.-M. Roth, From action to discourse: The bridging function of gestures, J. Cogn. Syst. Res. 3, 535 (2002).

[7] W.-M. Roth and D. V. Lawless, Scientific investigations, metaphorical gestures, and the emergence of abstract scientific concepts, Learn. Instr. 12, 285 (2002).

[8] P. Sengupta, K. D. Krinks, and D. B. Clark, Learning to deflect: Conceptual change in physics during digital game play, J. Learn. Sci. 24, 638 (2015).

[9] R. Lindgren, M. Tscholl, S. Wang, and E. Johnson, Enhancing learning and engagement through embodied interaction within a mixed reality simulation, Comput. Educ. 95, 174 (2016).

[10] E. Etkina, A. Karelina, M. Ruibal-Villasenor, D. Rosengrant, R. Jordan, and C. E. Hmelo-Silver, Design and reflection help students develop scientific abilities: Learning in Introductory Physics laboratories, J. Learn. Sci. 19, 54 (2010).

[11] E. Etkina, A. Van Heuvelen, S. White-Brahmia, D. Brookes, M. Gentile, S. Murthy, D. Rosengrant, and A. Warren, Scientific abilities and their assessment, Phys. Rev. ST Phys. Educ. Res. 2, 020103 (2006).

[12] W.-M. Roth, Gestures: Their role in teaching and learning, Rev. Educ. Res. 71, 365 (2001).

[13] J. M. Iverson and S. Goldin-Meadow, Why people gesture when they speak, Nature (London) 396, 228 (1998).

[14] S. Wagner Cook, T. K. Yip, and S. Goldin-Meadow, Gesturing makes memories that last, J. Mem. Lang. 63, 465 (2010).

[15] M. W. Alibali and M. J. Nathan, Embodiment in mathematics teaching and learning: Evidence from learners' and teachers' gestures, J. Learn. Sci. 21, 247 (2012).

[16] W.-M. Roth and M. Welzel, From activity to gestures and scientific language, J. Res. Sci. Teach. 38, 103 (2001).

[17] S. Goldin-Meadow, How gesture promotes learning throughout childhood, Child Dev. Perspect. 3, 106 (2009).

[18] W.-M. Roth and D. V. Lawless, How does the body get into the mind?, Hum. Stud. 25, 333 (2002).

[19] W.-M. Roth, Thinking with hands, eyes, and signs: MultiModal science talk in a grade 6/7 unit on simple machines, Interact. Learn. Environ. 4, 170 (1994).

[20] J. Hindmarsh and C. Heath, Embodied reference: A study of deixis in workplace interaction, J. Pragmatics 32, 1855 (2000).

[21] E. Hutchins, How a cockpit remembers its speeds, Cogn. Sci. 19, 265 (1995).

[22] M. Alač, in Representation in Scientific Practice Revisited, edited by C. Coopmans, J. Vertesi, and M. E. Lynch (MIT Press, Cambridge, MA, 2014), pp. 61-87.

[23] G. Kress and T. van Leeuwen, Multimodal Discourse: The Modes and Media of Contemporary Communication (Edward Arnold, London, 2001).

[24] E. Hutchins, Cognition in the Wild (MIT Press, Cambridge, MA, 1995).

[25] E. Hutchins and S. Nomura, in Embodied Interaction: Language and Body in the Material World, edited by J. Streeck, C. Goodwin, and C. LeBaron (Cambridge University Press, New York, 2011), pp. 29-43.
[26] C. Goodwin, Action and embodiment within situated human interaction, J. Pragmatics 32, 1489 (2000).

[27] C. Goodwin, in Discourse, the Body, and Identity, edited by J. Coupland and R. Gwyn (Palgrave- MacMillan, New York, 2003), pp. 19-42.

[28] C. Goodwin, in Gesture, and the Dynamic Dimension of Language, edited by S. D. Duncan, J. Cassell, and E. T. Levy (John Benjamins Publishing Company, Amsterdam, 2007), pp. 195-212.

[29] E. Ochs, P. Gonzales, and S. Jacoby, in Interaction and Grammar, edited by E. Ochs, E. Schegloff, and S. Thompson (Cambridge University Press, Cambridge, England, 1996), pp. 328-369.

[30] O. Lindwall and G. Lymer, The dark matter of lab work: Illuminating the negotiation of disciplined perception in mechanics, J. Learn. Sci. 17, 180 (2008).

[31] T. Fredlund, J. Airey, and C. Linder, Exploring the role of physics representations: An illustrative example from students sharing knowledge about refraction, Eur. J. Phys. 33, 657 (2012).

[32] D. McNeill, Hand and Mind: What Gestures Reveal about Thought (University of Chicago Press, Chicago, 1992).

[33] E. T. Levy and D. McNeill, Speech, gesture, and discourse, Discourse Process. 15, 277 (1992).

[34] G. Holton and S. G. Brush, Physics, the Human Adventure (Rutgers University Press, New Brunswick, NJ, 2001).

[35] T.S. Kuhn, The Structure of Scientific Revolutions (University of Chicago Press, Chicago, 1970).

[36] N. G. Lederman, F. Abd-El-Khalick, R. L. Bell, and R. S. Schwartz, Views of nature of science questionnaire: Toward valid and meaningful assessment of learners' conceptions of nature of science, J. Res. Sci. Teach. 39, 497 (2002).

[37] D. Allchin, Lawson's shoehorn, or should the philosophy of science be rated "X"?, Sci. Educ. 12, 315 (2003).

[38] R. Harré, The Philosophies of Science (Oxford University Press, Oxford, 1985).

[39] E. Etkina and A. Van Heuvelen, in Research-Based Reform of University Physics, edited by E. F. Redish and P. Conney (American association of physics teachers, College Park, MD, 2007), pp. 1-48.

[40] E. Etkina, Students of physics-Listeners, observers, or collaborative participants in physics scientific practices?, Am. J. Phys. 83, 669 (2015).

[41] European Space Agency (ESA), Rosetta Mission, http://www.esa.int/Our_Activities/Space_Science/Rosetta, retrieved June 1, 2017.

[42] National Aeronautics and Space Administration (NASA), New horizons mission, https://www.nasa.gov/ mission_pages/newhorizons/main/, retrieved June 1, 2017.

[43] National Aeronautics and Space Administration (NASA), Kepler mission, https://www.nasa.gov/mission_pages/ kepler/main/index.html, retrieved June 1, 2017.

[44] K. Batygin and M.E. Brown, Evidence for a distant giant planet in the Solar System, Astron. J. 151, 22 (2016).

[45] B. Gregorcic, E. Etkina, and G. Planinsic, A new way of using the interactive whiteboard in a high school physics classroom: A case study, Res. Sci. Educ., DOI: 10.1007/ s11165-016-9576-0. 
[46] B. Gregorcic, Exploring Kepler's laws using an interactive whiteboard and Algodoo, Phys. Educ. 50, 511 (2015).

[47] B. Gregorcic and M. Bodin, Algodoo: A tool for encouraging creativity in physics teaching and learning, Phys. Teach. 55, 25 (2017).

[48] R. Lindgren and M. Johnson-Glenberg, Emboldened by embodiment: Six precepts for research on embodied learning and mixed reality, Educ. Res. 42, 445 (2013).

[49] H. Garfinkel, Studies in Ethnomethodology (Prentice- Hall, Englewood Cliffs, NJ, 1967).

[50] S. E. Clayman and J. Heritage, in Situated Order: Studies in the Organization of Talk and Embodied Activities, edited by P. Ten Have and G. Psathas (International Institute for Ethnomethodology and Conversation Analysis and University Press of America, Washington, DC, 1995), pp. 1-30.

[51] T. Van Leeuwen, Introducing Social Semiotics (Routledge, New York, 2005).

[52] J. Bezemer and D. Mavers, Multimodal transcription as academic practice: A social semiotic perspective, Int. J. Soc. Res. Methodol. 14, 191 (2011).

[53] D. Hammer and L. K. Berland, Confusing claims for data: A critique of common practices for presenting qualitative research on learning, J. Learn. Sci. 23, 37 (2014).
[54] A. Baldry and P. J. Thibault, Multimodal Transcription and Text Analysis (Equinox, London, 2006).

[55] A. B. Hostetter and M. W. Alibali, Language, gesture, action! A test of the gesture as simulated action framework, J. Mem. Lang. 63, 245 (2010).

[56] J. Bezemerand G. Kress, Writing in multimodal texts: A social semiotic account of designs for learning, Writ. Commun. 25, 166 (2008).

[57] S. Goldin-Meadow, Hearing Gesture: How Our Hands Help Us Think (Harvard University Press, Cambridge, MA, 2005).

[58] S. Goldin-Meadow, Talking and thinking with our hands, Curr. Dir. Psychol. Sci. 15, 34 (2006).

[59] W. Adams, Gesture with Interactive Computer Simulations, http://phet.colorado.edu/publications/Gesture.pdf, retrieved June 1, 2017.

[60] PhET Interactive Simulations, https://phet.colorado.edu/, retrieved June 1, 2017.

[61] The Universe and More-Educational Physics Games, http://www.theuniverseandmore.com, retrieved June 1, 2017.

[62] Test Tube Games, http://www.testtubegames.com, retrieved June 1, 2017 